\title{
INFLUÊNCIA DA FIBRA DE AÇO SOBRE A RESISTÊNCIA RESIDUAL À TRAÇÃO POR FLEXÃO OBTIDA SEGUNDO O RILEM TC 162-TDF (2002)
}

\section{Influence of steel fiber on residual resistance to flexion traction obtained by Rilem TC 162-TDF (2002)}

Fernando Vasconcelos Borba ${ }^{1}$, Jorcelan Pereira da Rocha ${ }^{2}$

Recebido em 20 de outubro de 2019; aceito em 15 de dezembro de 2019; disponível on-line em 31 de dezembro de 2019.



PALAVRAS CHAVE:

Fibra de aço;

Tensão residual;

Resistência à tração;

Flexão;

Reforço.

\section{KEYWORDS:}

Steel fiber;

Residual stress;

Tensile strength;

Flexure;

Reinforcement.

RESUMO: A caracterização de peças reforçadas com fibra de aço à tração pode ser realizada por meio de ensaios de tração direta, ou de compressão diametral ou de flexão. No que diz respeito aos ensaios de flexão, o RILEM TC 162-TDF (2002), além de descrever os procedimentos para a realização do referido ensaio, também avalia o comportamento à tração em termos das curvas carga x deslocamento e carga x CMOD (Crack Mouth Opening Displacement). 0 presente trabalho tensiona estudar as resistências residuais à tração na flexão $\left(f_{\text {ri }}\right)$. Nesse contexto, coletou-se da literatura um banco de dados formado por 46 ensaios de flexão em três pontos de prismas entalhados, será apresentado e avaliado, através de um estudo estatístico, para que propostas sejam estabelecidas para estimar as resistências residuais à tração na flexão $f_{\text {ri }}(i=1,2,3$ e 4). Além disso, também será analisado a capacidade destas propostas em reproduzir as relações tensão-CMOD e tensão-deslocamento, as quais são regularmente obtidas a partir do ensaio de flexão em três pontos de prismas entalhados. A análise dos resultados quanto à capacidade de estimar as resistências residuais obtidas em ensaios foi favorável. Evidencia-se o desempenho das estimativas de $f_{\mathrm{ri}}(i=1,2$ e 4$)$, com MED $\approx 1,0$ e CV $<25 \%$. A estimativa de $f_{r 3}$ foi mais dispersa, com Média $\approx 1,0$ e coeficiente de variação $>25 \%$, dado o elevado nível de fissuração do prisma no momento do registro dessa resistência. Por fim, este trabalho tem grande importância no meio cientifico principalmente, pois a fibra de aço é um material que vem sendo estudado a bastante tempo, e por se tratar de um material complexo, necessita-se um estudo prévio do comportamento da resistência á flexão de vigas reforçadas com fibra de aço, tendo como principal objetivo determinar valores $f_{\text {ri }}(i=1,2,3$ e 4$)$ de ensaio de flexão em três pontos de prismas entalhados.

ABSTRACT: The characterization of tensile steel fiber reinforced parts can be performed by direct tensile tests, diametral compression or flexural tests. With regard to flexural tests, the RILEM TC 162-TDF (2002), in addition to describing the procedures for performing this test, also evaluates the tensile behavior in terms of load $x$ displacement and load $x$ CMOD curves (Crack Mouth Opening Displacement). The present study stresses to study the residual tensile strengths in flexion $\left(f_{\text {ri }}\right)$. In this context, a database of 46 flexural tests on three notched prisms points will be collected from the literature. It will be presented and evaluated through a statistical study so that proposals can be established to estimate residual flexural tensile strengths. $\left(f_{\mathrm{ri}}\right)(\mathrm{i}=1,2,3$ and 4$)$. In addition, the ability of these proposals to reproduce the stress-CMOD and stress-displacement ratios, which are regularly obtained from the bending test on three notched prisms, will also be analyzed. The analysis of the results regarding the ability to estimate the residual resistances obtained in tests was favorable. The performance of the estimates of $\left(f_{\mathrm{ri}}\right)(\mathrm{i}=1,2$ and 4$)$ is evidenced, with MED $\approx 1.0$ and $C V<25 \%$. The estimate of $f_{\mathrm{r} 3}$ was more dispersed, with Mean $\approx 1.0$ and coefficient of variation $>25 \%$, given the high level of prism cracking at the time of recording of this resistance. Finally, this work is of great importance in the scientific environment, especially since steel fiber is a material that has been studied for a long time, and because it is a complex material, a previous study of the behavior of flexural strength is required. of steel fiber reinforced beams, with the main objective to determine values $\left(f_{\text {ri }}\right)(i=1,2,3$ and 4$)$ of bending test at three points of notched prisms.

\footnotetext{
* Contato com os autores:

${ }^{1}$ e-mail: fernando.ofc@hotmail.com ( F. V. Borba )

Engenheiro Civil, Mestrando em Estruturas e Construção Civil - UFPA - Universidade Federal do Pará.

2e-mail: jorcelan20@gmail.com ( J. P. ROCHA )

Eng. Civil, Pós graduando em Infraestrutura de transportes e rodovias - IPOG.
} 


\section{INTRODUÇÃO}

A evolução da engenharia civil é notória nos últimos anos e esse progresso pode ser constatado nos mais variados campos de aplicação. Nas considerações teóricas, por exemplo, tem-se o surgimento de novos conceitos e modelos mecânicos, nas questões de projeto, verificam-se os novos softwares de cálculo e no que diz respeito aos processos construtivos, comentam-se as inovações quanto às argamassas para assentamento de alvenarias e aos sistemas steel deck e bubbledeck. Além desses, outros incontáveis exemplos podem ser citados para outras ramificações da engenharia. Diante o inegável progresso, no que diz respeito aos materiais de construção, o concreto continua sendo o mais empregado a nível mundial.

De acordo com Carvalho (2015) a vantagem do concreto, com relação aos demais materiais de construção (aço, madeira, alumínio e outros), deve-se, entre inúmeros aspectos, à sua capacidade de ser moldado e ao processo de fabricação relativamente simples. Apesar da condição de destaque, o concreto apresenta o infortúnio de não resistir aos esforços de tração, havendo, desta maneira, a necessidade de complementá-lo com um reforço nestas regiões tracionadas. Atualmente, as armaduras (barras de aço) são os mecanismos de reforço mais empregados, caracterizando assim o concreto armado. Nesse cenário de reforço para o concreto, salienta-se também a utilização de fibras (concreto com fibras), uma prática antiga, mas com desenvolvimento tecnológico recente.

Para ENGENHARIA CONCRETA (2016) a aplicação de fibra no concreto está se desenvolvendo e tem por finalidade melhorar as características do material. Assim, as fibras melhoram a trabalhabilidade do concreto $e$ aumentam a capacidade estrutural após endurecido. Assim, com o crescimento das pesquisas e a inovação tecnológica já surgiram vários tipos de fibras para o concreto.
A caracterização de peças reforçadas com fibra de aço à tração pode ser realizada por meio de ensaios de tração direta, ou de compressão diametral ou de flexão. No que diz respeito aos ensaios de flexão, o RILEM TC 162-TDF (2002), além de descrever os procedimentos para a realização do referido ensaio, também avalia o comportamento à tração em termos das curvas carga $x$ deslocamento e carga $x$ CMOD (Crack Mouth Opening Displacement). As resistências residuais à tração por flexão, $f_{\text {ri, }}$ são parâmetros obtidos experimentalmente com base nas curvas carga $x$ deslocamento ou carga $\times C M O D$ e conforme sugere as recomendações do RILEM TC 162-TDF (2002), são comumente utilizados no dimensionamento à flexão e ao corte de peças reforçadas com fibras de aço.

\subsection{CONCRETO REFORÇADO COM FIBRA DE AÇO (CRFA)}

Para Chaudhary et al., (2017) a fibra de aço é uma espécie de material compósito avançado, que é mais amplamente utilizado para reforço de concreto na construção e trabalho de engenharia hoje em dia. As fibras de aço, geralmente usado em concreto, são feitas de aço carbono e são fabricadas em várias formas e tamanhos, Figura 1. Fibras de aço misturado no concreto pode fornecer uma alternativa para o fornecimento de barras de aço convencionais. O seu uso no concreto pode melhorar suas muitas propriedades. As primeiras dosagens do Concreto Reforçado com Fibras de Aço (CRFA) datam da década de setenta e continham matrizes cimentícias compostas de agregados graúdos e fibras metálicas lisas. Inicialmente as fibras de aço utilizadas apresentavam resistências entre 500 e $900 \mathrm{MPa}$ e geometrias cilíndricas. Sabia-se à época que seções com maior área de contato entre fibra e matriz aumentariam o desempenho do compósito, mas devido limitações tecnológicas, era inexequível desenvolver fibras com outras seções transversais. Posteriormente, com a adoção de novas tecnologias, foi possível desenvolver-se fibras de aço com novas seções transversais. 


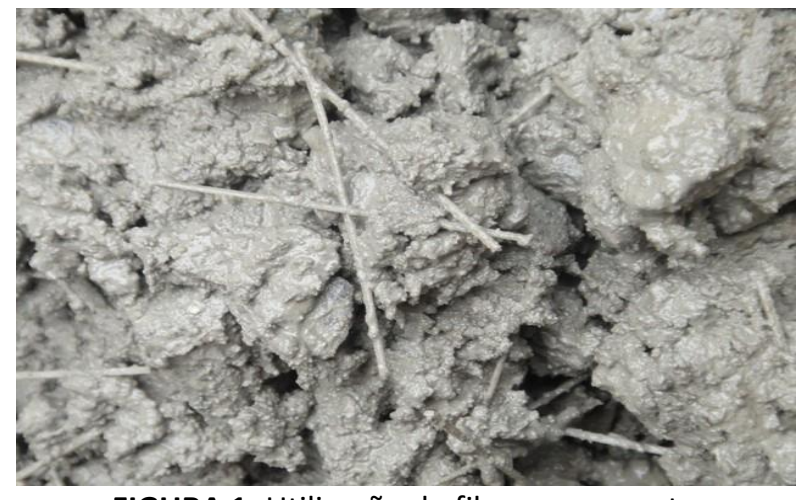

FIGURA 1: Utilização de fibra no concreto. FONTE: ENGENHARIA CONCRETA (2016).

\subsection{PREPARO DO CONCRETO REFORÇADO COM FIBRAS DE AÇO}

$\mathrm{Na}$ mistura, o lançamento e o adensamento do concreto com fibras de aço. $O$ processo de produção deve garantir uma dispersão uniforme das fibras e trabalhabilidade suficiente no estado fresco. Embora uma maior razão de aspecto melhore as propriedades do concreto endurecido, esta dificulta a trabalhabilidade no estado fresco. Em resumo, pode-se afirmar que o aumento no comprimento e no volume das fibras dificulta a trabalhabilidade e a dispersão uniforme. Mesmo para fibras de comprimentos pequenos (12-50 mm), apenas $0,5 \%$ a $1,5 \%$ de fibras podem ser utilizadas com processos convencionais de mistura. Conteúdos de fibras acima de $2 \%$ geralmente resultam em pobre trabalhabilidade e dispersão das fibras (KANG, 2011).

\subsubsection{Lançamento das fibras}

Há diversos estudos sobre o quanto interfere a orientação e lançamento das fibras. Fazendo com que diversos pesquisadores trabalhem num coeficiente através dos estudos propostos pelos mesmos. KANG (2011), as condições de produção do concreto com fibras afetam a distribuição e orientação das fibras na matriz no concreto. As fibras podem não proporcionar um reforço uniforme com a mesma eficiência em todas as direções. Na maioria dos casos, as orientações na matriz do concreto ocorrem devido ao resultado de vários fatores: a propriedades de estado fresco, lançamento, a geometria da fôrma, do tipo de vibração e o método de produção.

Conforme Kang et al., (2011), deve-se notar que as características de distribuição de fibras são geralmente dependentes da direção de lançamento do concreto. Por isso, em seu estudo os autores verificaram a influência da forma de lançamento do CRFA nos moldes, mais precisamente na direção de colocação e as características de distribuição das fibras em relação às propriedades mecânicas de concretos de ultra resistência reforçados com fibras de aço (steel fibre-reinforced ultra high strength concrete - SFRUHSC). A preparação das amostras se deu de duas formas, uma por lançar o material paralelamente à direção longitudinal da forma (amostras - PL), e outra por lançar o material transversalmente à direção longitudinal da forma (amostras - TL), exibido nas Figuras 2 e 3 . Ambas as amostras foram executadas com proporção de: cimento 1 ; areia 1,1; filler 0,3 ; sílica 0,25 ; água 0,25 ; superplastificante 0,018 e fibra de aço $2 \%$.

(a)

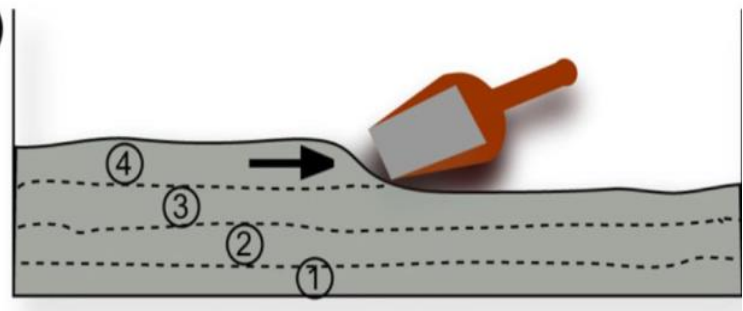

FIGURA 2: Preparação das amostras: Lançamento longitudinal (PL)

FONTE: Kang et al., (2011)

(b)



FIGURA 3: Preparação das amostras: Lançamento Transversal (TL)

FONTE: Kang et al., (2011)

\subsubsection{Tipos de fibras}

Para ENGENHARIA CONCRETA (2016), os diversos tipos de fibras podem ser incorporados à 
matriz cimentícia, a depender das propriedades que se deseja melhorar: ductilidade, resistência à tração, tenacidade, características de deformação, dentre outros. Dentre elas podem ser citadas, as fibras poliméricas. - polipropileno, polietileno e poliéster; metálicas - aço; vegetais - coco, piaçava, sisal e celulose, normalmente empregada na produção de componentes como tijolos, telhas e cochos; e minerais - carbono, amianto e vidro, como exibido na Figura 4.

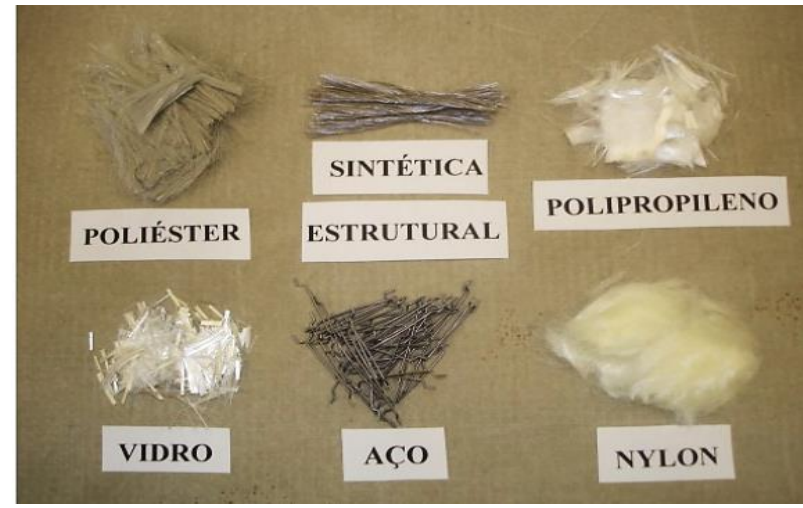

FIGURA 4: Fibras para concreto.

FONTE: Fibras para concreto

(https://www.cimentoitambe.com.br/fibras-para-concreto/)

\subsection{Estudos prévios}

Cunha (2003) afirma que os códigos de projeto e orientações para concreto reforçado com fibra (FRC) propõem diferentes modelos constitutivos que compartilham uma abordagem comum: os parâmetros que determinam o comportamento à tração são recuperados a partir dos resultados de ensaios de flexão em vigas. A estrutura e a configuração de carga adotada produzem uma evolução gradual de liberação de energia durante o processo de carregamento, o que simplifica o controle e a avaliação da resposta do material. Além disso, uma configuração isostática é usada, o que significa que as forças internas podem ser facilmente determinadas.

RILEM TC 162-TDF (2002) descreve os procedimentos para a realização do ensaio de flexão em três pontos com vigas entalhadas, propondo uma avaliação através do comportamento dos parâmetros (carga $\mathrm{X}$ deslocamento) e [carga CMOD (Crack
Mouth Opening Displacement)]. Para a obtenção da resistência equivalente de tração na flexão, tornase necessário medir o deslocamento, porém para a obtenção das tensões residuais, $f_{\text {rì }}$ é possível utilizar qualquer um dos dois parâmetros: (carga $X$ deslocamento) ou (carga X CMOD), havendo, portanto, uma correlação entre o deslocamento $(\delta)$ e o CMOD.

Critérios de dimensionamento, tais como a determinação do gráfico $\sigma-\varepsilon$, são tratados pelo RILEM TC 162-TDF (2002). Este documento traz orientações para dimensionamento à flexão e ao cisalhamento de peças reforçadas com fibras de aço, contudo a obtenção dos valores de $f_{\text {ri }}$ são feitos através de ensaios experimentais, dificultando assim aplicação do método. Parâmetros importantes como a resistência à tração do concreto, o consumo de fibras, a resistência das fibras e o fator deforma não são levados em consideração para o dimensionamento.

\section{OBJETIVO}

Com o objetivo de apresentar propostas para obtenção das tensões residuais através de características do concreto reforçado com fibra de aço sem a necessidade de ensaios experimentais, este trabalho apresenta um estudo empírico através de um banco de dados que correlacione os parâmetros: consumo de fibras (Cf), Fator de forma (ff) com as tensões residuais.

\section{METODOLOGIA}

O presente trabalho estudou as resistências residuais à tração na flexão $\left(f_{\text {ri }}\right)$. Nesse contexto, coletou-se da literatura um banco de dados formado por 46 ensaios de flexão em três pontos de prismas entalhados, conforme sugere o ModelCode10 (2011), será apresentado e avaliado, através de um estudo estatístico, para que propostas sejam estabelecidas para estimar as resistências residuais à tração na flexão $f_{\text {ri }}(i=1$, 2, 3 e 4). Além disso, se analisa a capacidade 
destas propostas em reproduzir as relações tensão-CMOD e tensão-deslocamento, as quais são regularmente obtidas a partir do ensaio de flexão em três pontos de prismas entalhados.

\subsection{RESULTADOS E DISCUSSÕES}

O banco de dados utilizado contou com 46 ensaios de flexão de vigas entalhadas. Todas as vigas foram reforçadas apenas com fibras de aço do tipo "hooked-end" e apresentaram os principais parâmetros variando a seguinte forma:

a. Fator de forma (If/df): $45-95,24$

b. Consumo de fibras (\%): 0,13-0,93

A Figura 5 (a) e 5 (b) e a Equação 1 exemplificam $\circ$ procedimento utilizado para estabelecer os valores de $f_{r i}$

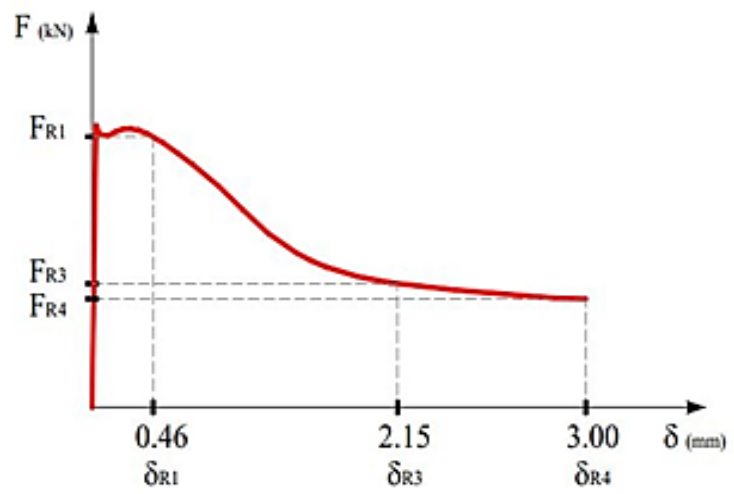

a) Carga $x$ Deslocamento

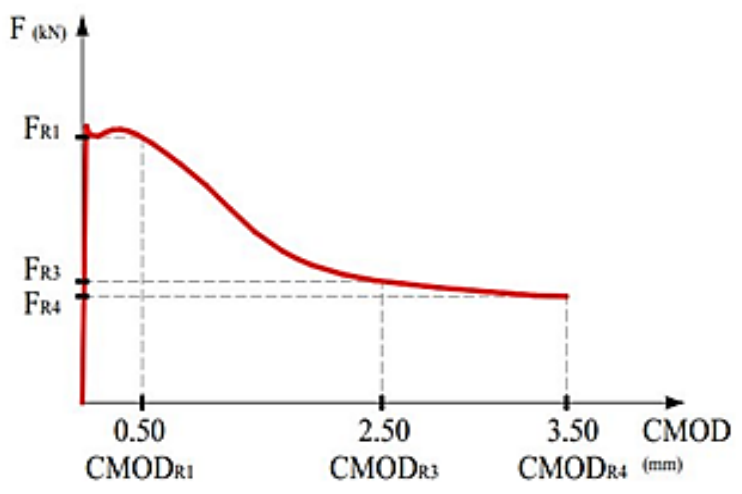

b) Carga $\times \mathrm{CMOD}$

FIGURA 5: Procedimento para estabelecer os valores. FONTE: RILEM TC 162-TDF (2002)

Os valores de $f_{\text {ri }}$ foram obtidos através das curvas [(carga $X$ deslocamento) ou (carga $X$
CMOD)], de acordo com as orientações do RILEM TC 162-TDF (2002) e podem ser calculados através da Equação 1:

$$
f_{r i}=\frac{3}{2} \frac{F_{R i} L}{b\left(h_{s p}\right)^{2}} M P a
$$

Sendo: $f_{\mathrm{ri}}$ o valor da carga correspondente à $\delta R i$ ou COMODi, em Newtons, $L$, $b$ e $h_{s p}$ sendo o vão entre apoio, largura da viga e altura o vão entre os apoios e altura acima do entalhe, em $\mathrm{mm}$. $O f_{\text {ri }}$ é calculado em MPa na Tabela 1.

\subsection{ANÁLISE DOS PARÂMETROS}

A análise dos dados inicialmente consistiu em verificar os parâmetros que influenciam os $f_{\mathrm{ri}}$ e estabelecer linhas de tendência que as representassem. Os parâmetros selecionados foram: Consumo de Fibras e Fator de Forma. Na sequência, equações que relacionam esses parâmetros foram analisadas com o objetivo se julga-las diretamente ou inversamente proporcionais e posteriormente inserir os termos preponderantes nas equações de acordo com a afinidade deles com as tensões residuais a serem representadas, a inserção desses termos, acabam melhorando a relação com os $f_{\text {ri }}$ e criando a formula de acordo a regressão univariada de potência, o qual obteve melhores resultados, o critério foi avaliar o nível de conservadorismo e dispersão de acordo com a Equação 2.

$$
\lambda \mathrm{i}=f_{r i}, \mathrm{EXP} / f_{r i}, \text { TEO }
$$

A qual representa a relação entre os valores de resistência obtidos experimentalmente e teoricamente. Os valores de $\lambda i$ foram avaliados segundo uma adaptação do critério de penalidade, Tabela 2. 
TABELA 1: Banco de Dados.

\begin{tabular}{|c|c|c|c|c|c|c|c|c|c|}
\hline Autor & Peça & $\begin{array}{l}\text { Tipo de } \\
\text { fibra }\end{array}$ & \begin{tabular}{|c|} 
Volume \\
de Fibra \\
$(\%)$
\end{tabular} & $\mid f / d f$ & $v f .(I f / d f)$ & $\begin{array}{l}f r 1 \\
\mathrm{MPa}\end{array}$ & $\begin{array}{l}f r 2 \\
\mathrm{MPa}\end{array}$ & $\begin{array}{l}f r 3 \\
\mathrm{MPa}\end{array}$ & $\begin{array}{c}\text { fr4 } \\
\text { Mpa }\end{array}$ \\
\hline $\begin{array}{l}\text { Poh et al } \\
\text { (2008) }\end{array}$ & A & Hooked & 0,40 & 80,00 & 0,32 & 4,00 & 3,40 & 2,60 & 2,10 \\
\hline \multirow{4}{*}{ Barros (2003) } & S2_3 & Hooked & 0,33 & 65,22 & 0,22 & 3,68 & 3,92 & 3,2 & 3,04 \\
\hline & S2_4 & Hooked & 0,47 & 65,22 & 0,31 & 3,68 & 5,12 & 3,2 & 3,52 \\
\hline & S2_5 & Hooked & 0,67 & 65,22 & 0,44 & 6,88 & 7,04 & 7,12 & 6,88 \\
\hline & S2_6 & Hooked & 0,93 & 65,22 & 0,61 & 7,28 & 10,24 & 9,92 & 6,64 \\
\hline $\begin{array}{c}\text { Pujadas et al } \\
\text { (2014) }\end{array}$ & $A v-S F 2$ & Hooked & 0,80 & 80,65 & 0,65 & 6,40 & 6,12 & 6,24 & 6,47 \\
\hline $\begin{array}{l}\text { Pujadas et al } \\
\text { (2012) }\end{array}$ & SF1_25 & Hooked & 0,25 & 63,64 & 0,16 & 2,55 & 2,44 & 2,22 & 1,99 \\
\hline \multirow{7}{*}{$\begin{array}{l}\text { Tiberti et al } \\
\text { (2014) }\end{array}$} & 1_0.50M & Hooked & 0,50 & 48,39 & 0,24 & 3,40 & 3,40 & 3,10 & 2,50 \\
\hline & 2_0.50M & Hooked & 0,50 & 48,39 & 0,24 & 3,55 & 3,60 & 3,00 & 2,20 \\
\hline & 3_0.50M & Hooked & 0,50 & 48,39 & 0,24 & 3,60 & 3,70 & 2,90 & 2,20 \\
\hline & 4_0.50M & Hooked & 0,50 & 48,39 & 0,24 & 3,70 & 3,90 & 3,20 & 2,60 \\
\hline & 5_0.50M & Hooked & 0,50 & 48,39 & 0,24 & 4,15 & 4,05 & 3,35 & 2,80 \\
\hline & $6 \_0.50 \mathrm{M}$ & Hooked & 0,50 & 48,39 & 0,24 & 4,95 & 4,30 & 3,80 & 3,00 \\
\hline & 7_0.50M & Hooked & 0,50 & 48,39 & 0,24 & 5,50 & 5,40 & 4,15 & 3,50 \\
\hline $\begin{array}{l}\text { Amin et } \\
\text { al(2016) }\end{array}$ & B50-0-0-0 & Hooked & 0,67 & 66,67 & 0,45 & 5,12 & 7,22 & 6,72 & 6,40 \\
\hline \multirow{2}{*}{$\begin{array}{l}\text { Amin et al } \\
\text { (2015) }\end{array}$} & AM-0,4-EH & Hooked & 0,40 & 63,64 & 0,25 & 4,96 & 4,33 & 3,61 & 3,04 \\
\hline & AM-0,8-EH & Hooked & 0,80 & 63,64 & 0,51 & 7,36 & 7,44 & 5,87 & 4,85 \\
\hline \multirow{6}{*}{$\begin{array}{l}\text { Cuenca et al } \\
\text { (2015) }\end{array}$} & $\mathrm{M}-45 / 50 \mathrm{BN}$ & Hooked & 0,67 & 45,00 & 0,30 & 4,18 & 4,75 & 4,43 & 4,17 \\
\hline & $\mathrm{M}-65 / 40 \mathrm{BN}$ & Hooked & 0,67 & 65,00 & 0,44 & 6,83 & 7,49 & 6,03 & 5,06 \\
\hline & $\mathrm{M}-80 / 50 \mathrm{BN}$ & Hooked & 0,67 & 80,00 & 0,54 & 7,42 & 7,60 & 6,90 & 6,46 \\
\hline & $\mathrm{M}-80 / 30 \mathrm{BP}$ & Hooked & 0,67 & 80,00 & 0,54 & 6,93 & 8,09 & 7,13 & 6,13 \\
\hline & $\mathrm{M}-80 / 40 \mathrm{BP}$ & Hooked & 0,67 & 80,00 & 0,54 & 7,71 & 8,80 & 8,19 & 7,56 \\
\hline & $\mathrm{H}-45 / 50 \mathrm{BN}$ & Hooked & 0,67 & 45,00 & 0,30 & 7,28 & 7,44 & 5,94 & 4,85 \\
\hline \multirow{3}{*}{$\begin{array}{l}\text { Lee et al } \\
(2010)\end{array}$} & C45V250 & Hooked & 0,25 & 95,24 & 0,24 & 4,33 & 3,03 & 2,23 & 1,61 \\
\hline & C45V375 & Hooked & 0,375 & 95,24 & 0,36 & 4,65 & 3,52 & 2,69 & 2,38 \\
\hline & C45V500 & Hooked & 0,50 & 95,24 & 0,48 & 6,18 & 5,00 & 3,40 & 2,72 \\
\hline Moraes (2013) & Vf60fc70 & Hooked & 0,80 & 67,27 & 0,54 & 9,06 & 9,58 & 8,08 & 6,96 \\
\hline \multirow{8}{*}{ Cunha (2003) } & F65/60Qf15Cv0ld7 & Hooked & 0,20 & 67,00 & 0,13 & 2,14 & 1,98 & 1,92 & 1,89 \\
\hline & F65/60Qf35Cv0ld7 & Hooked & 0,47 & 67,00 & 0,31 & 3,20 & 3,01 & 2,62 & 2,50 \\
\hline & F65/60Qf15Cv0ld28 & Hooked & 0,20 & 67,00 & 0,13 & 2,56 & 2,43 & 2,42 & 2,40 \\
\hline & F65/60Qf25Cv0ld28 & Hooked & 0,33 & 67,00 & 0,22 & 4,13 & 3,65 & 3,36 & 3,20 \\
\hline & F65/60Qf35Cv0ld28 & Hooked & 0,47 & 67,00 & 0,31 & 3,84 & 3,84 & 3,81 & 3,71 \\
\hline & F65/60Qf45Cv0ld28 & Hooked & 0,60 & 67,00 & 0,40 & 5,86 & 6,02 & 5,52 & 5,10 \\
\hline & F65/60Qf15Cv0ld90 & Hooked & 0,20 & 67,00 & 0,13 & 2,43 & 2,48 & 2,40 & 2,30 \\
\hline & F65/60Qf25Cv0ld90 & Hooked & 0,33 & 67,00 & 0,22 & 4,10 & 3,58 & 3,36 & 3,23 \\
\hline
\end{tabular}




\begin{tabular}{|c|c|c|c|c|c|c|c|c|c|}
\hline \multirow[t]{2}{*}{ Autor } & \multirow[t]{2}{*}{ Peça } & \multirow{2}{*}{$\begin{array}{l}\text { Tipo de } \\
\text { fibra }\end{array}$} & $\begin{array}{l}\text { Volume } \\
\text { de Fibra }\end{array}$ & \multirow[t]{2}{*}{ If/df } & \multirow[t]{2}{*}{$v f .(I f / d f)$} & \multirow{2}{*}{$\begin{array}{c}f r 1 \\
\mathrm{MPa} \\
\end{array}$} & \multirow{2}{*}{$\begin{array}{c}f r 2 \\
\mathrm{MPa} \\
\end{array}$} & \multirow{2}{*}{$\begin{array}{c}f r 3 \\
\mathrm{MPa}\end{array}$} & \multirow{2}{*}{$\begin{array}{l}\text { fr4 } \\
\text { Mpa }\end{array}$} \\
\hline & & & (\%) & & & & & & \\
\hline \multirow{8}{*}{ Cunha (2003) } & F65/60Qf35Cv0ld90 & Hooked & 0,47 & 67,00 & 0,31 & 3,84 & 3,86 & 3,76 & 3,70 \\
\hline & F65/60Qf45Cv0ld90 & Hooked & 0,60 & 67,00 & 0,40 & 5,84 & 5,98 & 5,50 & 5,12 \\
\hline & F80/60Qf10Cv0ld7 & Hooked & 0,13 & 80,00 & 0,10 & 1,44 & 1,44 & 1,38 & 1,30 \\
\hline & F80/60Qf20Cv0ld7 & Hooked & 0,27 & 80,00 & 0,22 & 2,45 & 2,38 & 2,26 & 2,22 \\
\hline & F80/60Qf10Cv0ld28 & Hooked & 0,13 & 80,00 & 0,10 & 1,76 & 1,65 & 1,60 & 1,44 \\
\hline & F80/60Qf20Cv0ld28 & Hooked & 0,27 & 80,00 & 0,22 & 2,98 & 2,64 & 2,40 & 2,26 \\
\hline & F80/60Qf10Cv0ld90 & Hooked & 0,13 & 80,00 & 0,10 & 1,68 & 1,60 & 1,66 & 1,44 \\
\hline & F80/60Qf20Cv0ld90 & Hooked & 0,27 & 80,00 & 0,22 & 2,62 & 2,38 & 2,14 & 1,92 \\
\hline \multirow{3}{*}{ Lima (2012) } & P1 & Hooked & 0,26 & 80,00 & 0,21 & 3,14 & 2,52 & 1,80 & 1,47 \\
\hline & P2 & Hooked & 0,52 & 80,00 & 0,42 & 7,51 & 6,94 & 6,14 & 5,10 \\
\hline & P3 & Hooked & 0,78 & 80,00 & 0,62 & 7,00 & 6,73 & 5,27 & 4,36 \\
\hline
\end{tabular}

TABELA 02: Critério de Collins

\begin{tabular}{|c|c|c|}
\hline Intervalo $\boldsymbol{\lambda} \boldsymbol{i}$ & Classificação & Penalidade \\
\hline $0,5>$ & Extremamente Perigoso & 10 \\
\hline$[0,5-0.85[$ & Perigoso & 5 \\
\hline$[0.85-1,15[$ & Segurança Apropriada & 0 \\
\hline$[1,15-2,00[$ & Conservador & 1 \\
\hline$>2,00$ & Extremamente Conservador & 2 \\
\hline
\end{tabular}

Fonte: Collins, M.P. (2001).

Fundamentado nos resultados coletados a partir do banco de dados apresentado neste trabalho e nos referidos autores apresentados na Tabela 1 , verificouse, tomando como referência a resistência residual $f_{r i}$, (Figuras 6 a 9), que o fator de forma e volume de fibras de reforço do CRFA, são parâmetros importantes na avaliação da resistência residual $f_{r i}$ e, de um modo mais geral, no comportamento fissurado do concreto com fibras. Além disso, a Figura 6 também permite confirmar que a relação $v f .(I f / d f)$ se mostra mais expressiva, tendo em vista que quanto mais o valor se aproxima de um, melhor o comportamento às oscilações das resistências $f_{r i}(i=1,2,3$ e 4). 


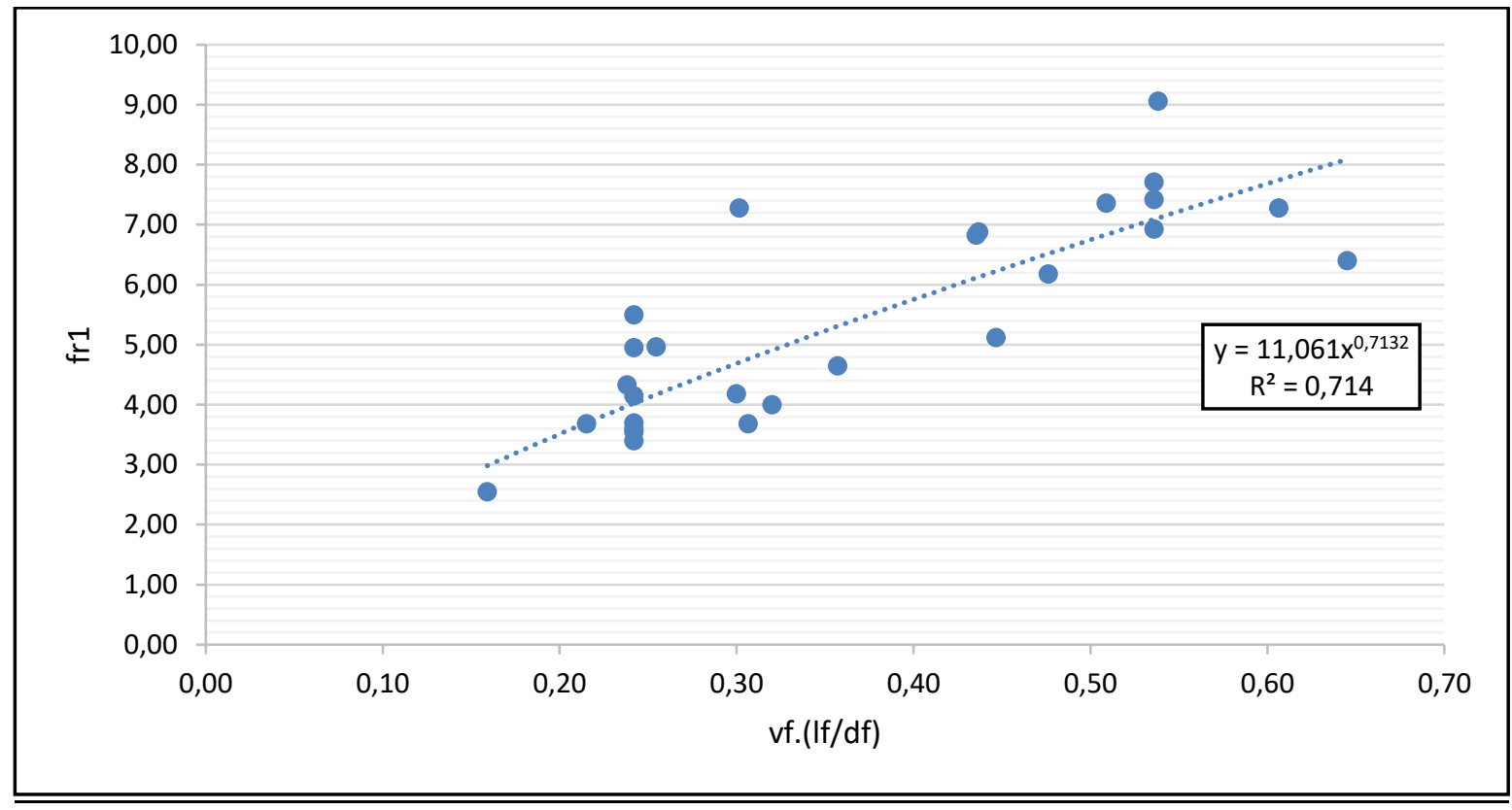

FIGURA 6: Relações entre os parâmetros analisados para o comportamento softening. Fonte: Elaborada pelos autores.

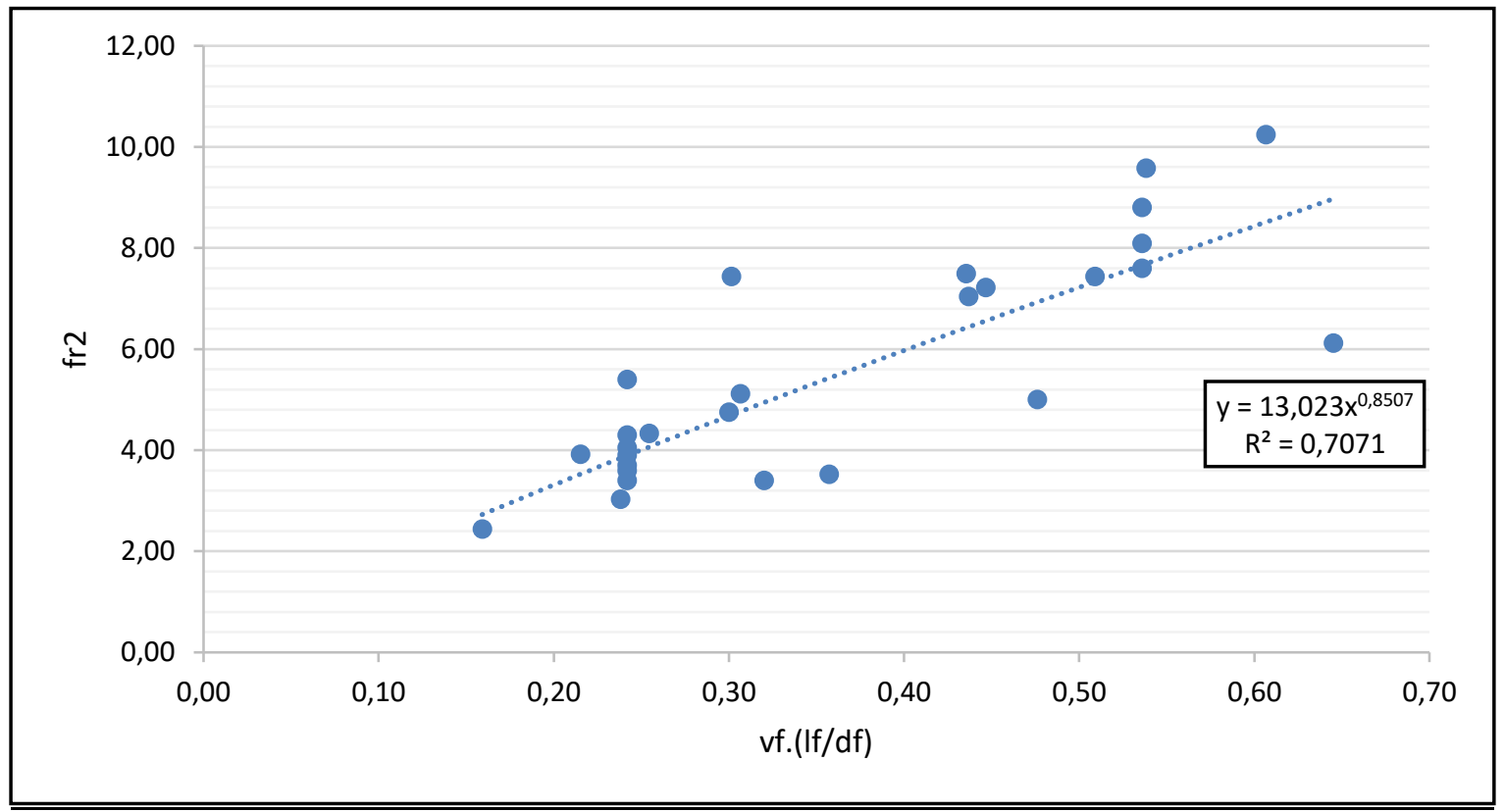

FIGURA 7: Relações entre os parâmetros analisados para o comportamento softening Fonte: Elaborada pelos autores. 




FIGURA 8: Relações entre os parâmetros analisados para o comportamento softening Fonte: Elaborada pelos autores.

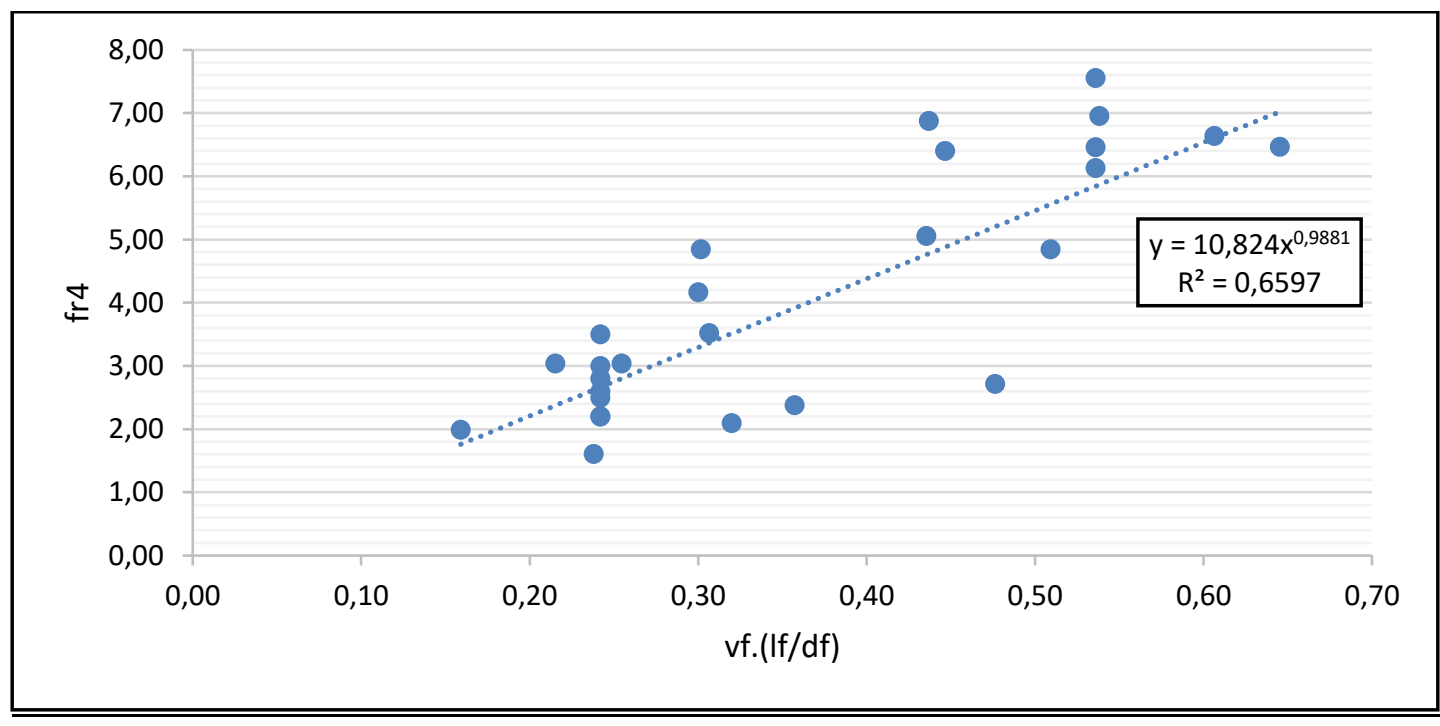

FIGURA 9: Relações entre os parâmetros analisados para o comportamento softening Fonte: Elaborada pelos autores.

$$
\begin{aligned}
& F_{R 1}=10,3 x\left(v_{f} x \frac{l f}{d f}\right)^{0,8} \\
& F_{R 2}=10,5 x\left(v_{f} x \frac{l f}{d f}\right)^{0,8} \\
& F_{R 3}=8,89 x\left(v_{f} x \frac{l f}{d f}\right)^{0,8} \\
& F_{R 4}=7,69 x\left(v_{f} x \frac{l f}{d f}\right)^{0,8}
\end{aligned}
$$


A análise das Equações $x$ a y foi conduzida avaliando-se o comportamento do parâmetro $\lambda \mathrm{i}$ quanto à dispersão dos resultados e quanto ao critério apresentado na Tabela 1. Os resultados das análises são apresentados nas Figuras 10, 11 e 12, onde se verifica a dispersão das respostas, a porcentagem de resultados seguros $(\lambda i \geq 1)$ e a curva "box and whiskers". Por fim, a Tabela 3 apresenta $\circ$ resumo estatístico da análise.

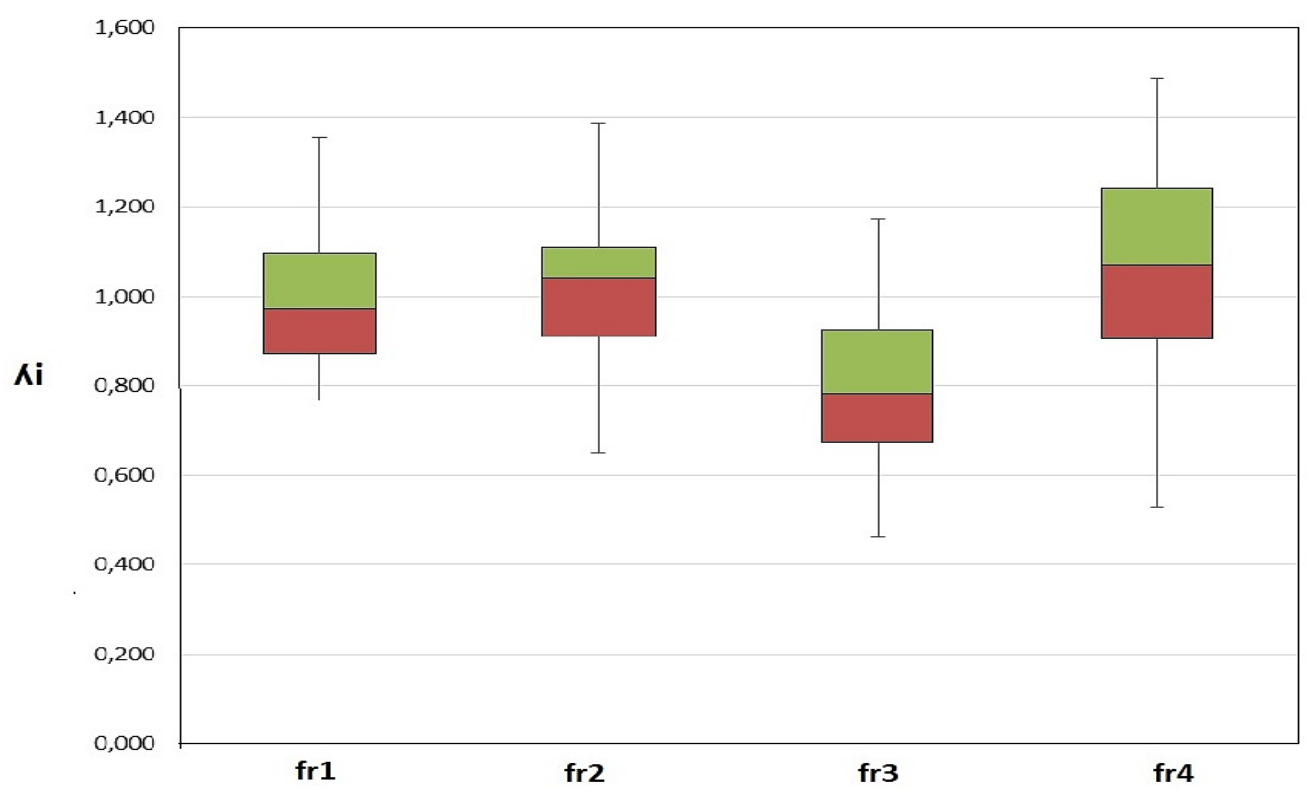

FIGURA 10: Curva box and whiskers - Resistência.

Fonte: Elaborada pelos autores.

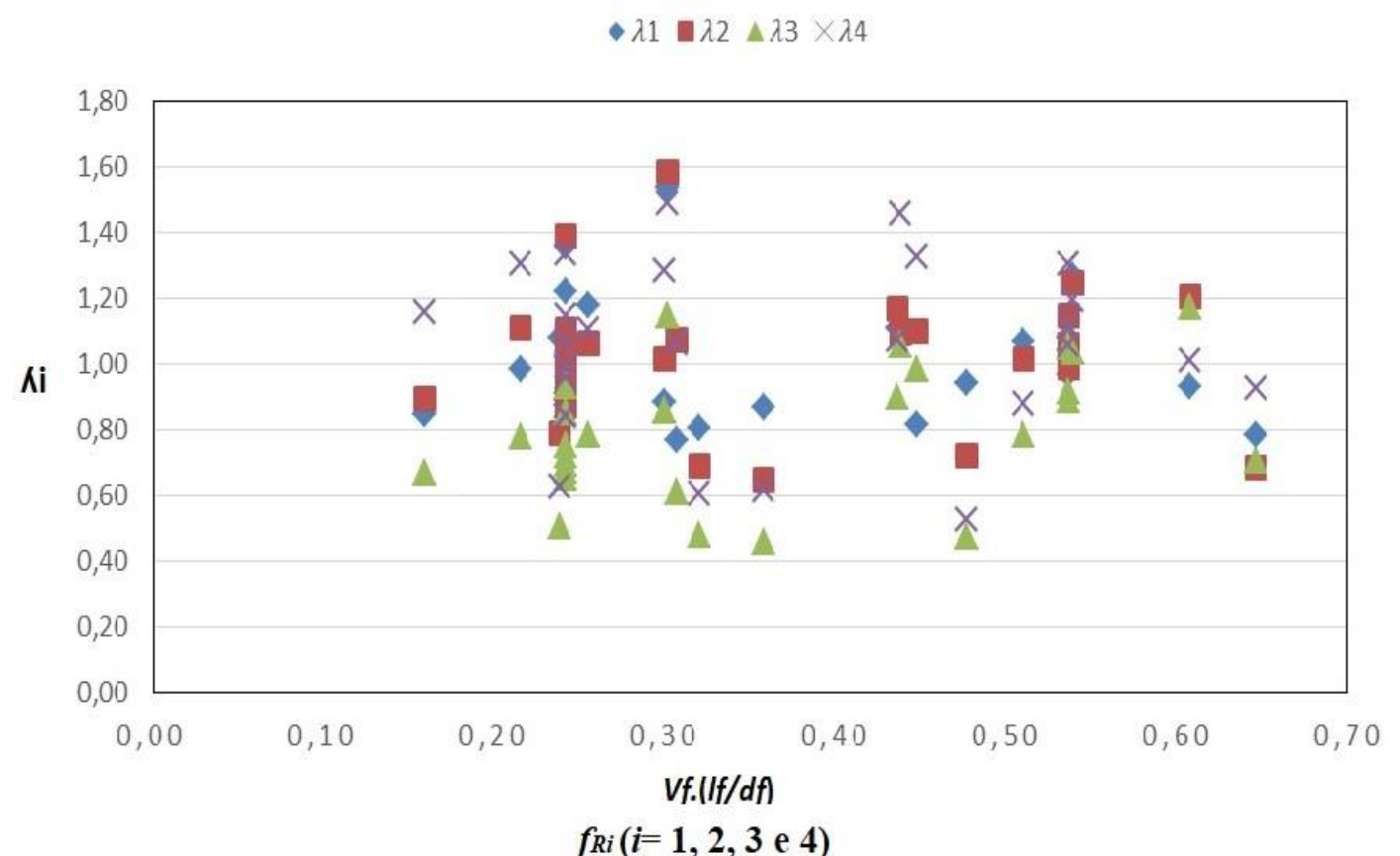

FIGURA 11: Dispersão das respostas.

Fonte: Elaborada pelos autores. 


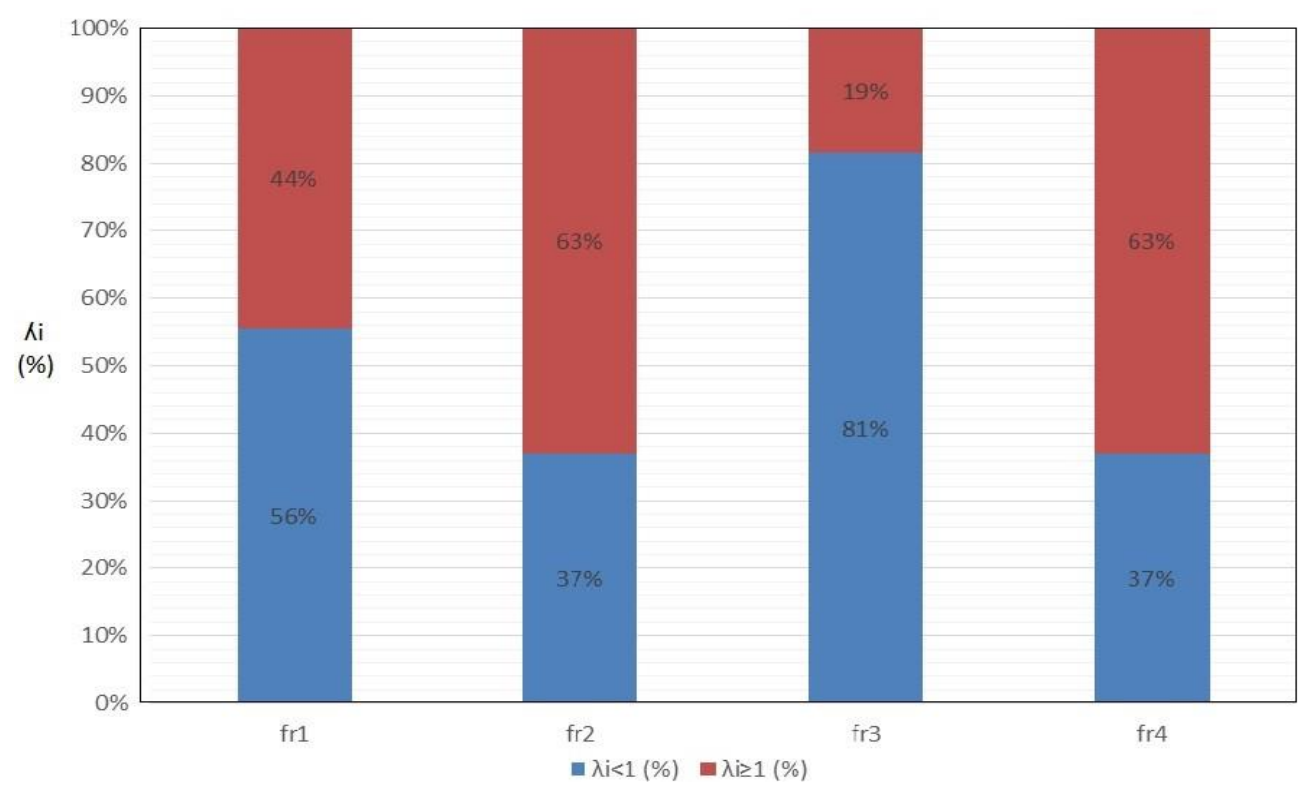

FIGURA 12: Resultados seguros em porcentagem.

Fonte: Elaborada pelos autores.

\begin{tabular}{|c|c|c|c|c|c|c|c|c|}
\hline \multirow{2}{*}{$\begin{array}{c}\text { Parâmetro } \\
\lambda 1\end{array}$} & \multicolumn{2}{|c|}{ fr1 } & \multicolumn{2}{|c|}{ fr2 } & \multicolumn{2}{|c|}{ fr3 } & \multicolumn{2}{|c|}{ fr4 } \\
\hline & $\begin{array}{l}\text { no de } \\
\text { peças }\end{array}$ & Penal; & $\begin{array}{l}\text { no de } \\
\text { peças }\end{array}$ & Penal; & $\begin{array}{l}\text { no de } \\
\text { peças }\end{array}$ & Penal; & $\begin{array}{l}\text { no de } \\
\text { peças }\end{array}$ & Penal; \\
\hline$<0,50$ & 0 & 0 & 0 & 0 & 3 & 30 & 0 & 0 \\
\hline$[0,50-0,85]$ & 6 & 30 & 5 & 25 & 12 & 60 & 6 & 30 \\
\hline$[0,85-1,15]$ & 16 & 0 & 16 & 0 & 11 & 0 & 12 & 0 \\
\hline$[1,15-2]$ & 5 & 5 & 6 & 6 & 1 & 1 & 9 & 9 \\
\hline$\geq 2$ & 0 & 0 & 0 & 0 & 0 & 0 & 0 & 0 \\
\hline Total & 27 & 35 & 27 & 31 & 27 & 91 & 27 & 39 \\
\hline Média & \multicolumn{2}{|c|}{1,01} & \multicolumn{2}{|c|}{1,02} & \multicolumn{2}{|c|}{0,80} & \multicolumn{2}{|c|}{1,05} \\
\hline $\begin{array}{l}\text { Desvio } \\
\text { padrão }\end{array}$ & \multicolumn{2}{|c|}{0,19} & \multicolumn{2}{|c|}{0,21} & \multicolumn{2}{|c|}{0,20} & \multicolumn{2}{|c|}{0,26} \\
\hline CV & \multicolumn{2}{|c|}{$18,502 \%$} & \multicolumn{2}{|c|}{$20,737 \%$} & \multicolumn{2}{|c|}{$25,329 \%$} & \multicolumn{2}{|c|}{$24,619 \%$} \\
\hline
\end{tabular}

Fonte: Collins, M.P. (2001)

Os resultados apresentados na Tabela 3 a partir das Equações (3) à (6), mostram que os valores de $f_{R}, i=(1,2,3,4)$ apresentam-se conservadores a favor da segurança e baixas penalidades estabelecidas pelo critério de Collins (2001). 
Funções potencias foram adotadas na formulação das propostas para $f_{r i}(i=1,2,3$ e 4). Para assegurar a praticidade dessas propostas, apenas parâmetros de simples avaliação foram ponderados na fase atual do estudo.

As equações obtidas de forma empírica relacionam os valores de resistência à compressão do concreto, fator de forma e consumo de fibras. Após a análise estatística percebe-se que os parâmetros citados anteriormente têm uma influência significativa nas resistências residuais $f_{R}, i=(1,2,3,4)$. Os resultados apresentados a partir das Equações (3) à (6) mostra-se conservadores tanto quanto a penalidades, dispersão e segurança. Deve-se notar que os valores de $f_{R}, i=(3,4)$ estão um pouco dispersos e com coeficientes de variação altos. Portanto, pode-se ter uma estimativa conservadora quanto aos valores das resistências residuais através das Equações (3), (4), (5), (6).

\section{CONCLUSÕES}

A análise dos resultados quanto à capacidade de estimar as resistências residuais obtidas em ensaios (ensaio de flexão em três pontos de prismas entalhados), foi favorável. Evidencia-se o desempenho das estimativas de $f_{r i}$ ( $i=1,2$ e 4), com MED $\approx 1,0$ e CV $<25 \%$. A estimativa de $f R 3$ foi mais dispersa, $M E D \approx 1,0$ e $C V$ $>25 \%$, dado o elevado nível de fissuração do prisma no momento do registro dessa resistência;

No que diz respeito ao desempenho das propostas em reproduzir a fase pós-pico das relações tensão-CMOD e tensão-deslocamento (ensaio de flexão em três pontos de prismas entalhados), destaca-se que estimativas válidas foram obtidas;

No que diz respeito à análise das propostas para $f_{r i}$, constatou-se que essas propostas foram aplicadas apropriadamente nos modelos de flexão do RILEM TC 162-TDF (2002). Salienta-se que a estimativa da resistência à flexão de vigas do banco de dados foi, em geral, acertada, com $M E D \approx 1,0$ e $C V<25 \%$;
Por fim, este trabalho tem grande importância no meio cientifico principalmente, pois a fibra de aço é um material que vem sendo estudado a bastante tempo, e por se tratar de um material complexo, necessita-se um estudo prévio do comportamento da resistência á flexão de vigas reforçadas com fibra de aço, tendo como principal objetivo determinar valores $f_{\mathrm{ri}},(i=1,2,3$ e 4) de ensaio de flexão em três pontos de prismas entalhados.

\section{REFERÊNCIAS BIBLIOGRÁFICAS}

AMIN, Ali.; FOSTER, Stephen J.; MITTONI, Aurelio. Derivation of the $\sigma-w$ relationship for SFRC from prism bending tests. KG, Berlin: Structural Concrete, v. 16, $p$. 93-105, 2015.

AMIN, Ali; FOSTER, Stephen J. Predicting the flexural response of steel fibre reinforced concrete prisms using a sectional model. New South Wales, Austrália: Cement and Concrete Composites, v. 67, p. 1-11, 2016.

BARROS, J.; MORAES, B.; MELO, G.; FRAZÃO, C. Assessment of the effectiveness of steel fibre reinforcement for the punching resistance of flat slabs by experimental research and design approach: Composites Part B, 2015.

CARVALHO, R.C; PINHEIRO, L.M. Cálculo e Detalhamento de Estruturas Usuais de Concreto Armado, v1. 1ed. São Paulo: Editora PINI Ltda, 2015.

CHAUDHARY, R.; AHAMAD, S.; PATEL, V.K; Nabiullah Khan.Experimental Analysis of Asbestos Fibre Reinforced Concrete Composite. Índia, 2017. Disponível em: $\quad<$ https://www.semanticscholar.org/paper/Experi mental-Analysis-of-Asbestos-Fibre-ReinforcedChaudhary-Ahamad/20aaa2a5b837bd2d2ffc49588d9 de38fd8a8decf>. doi: 10.9790/1684-1404021822. Acessado em 04/05/2019.

COLLINS, M.P. Evaluation of shear design procedures for concrete structures. A Report prepared for the CSA technical committee on reinforced concrete design, 2001.

CUECA, E.; ECHEGARAY-OVIEDO, J.; SERNA, P. Influence of concrete matrix and type of fiber on the shear behavior of self-compacting fiber reinforced concrete beams. Universitat Politècnica de València, Espanha, 2015.

CUNHA, V.; RIBEIRO, A.; BARROS, J; ANTUNES, A. Betão Reforçado com Fibras de Aço: recomendações normativas, investigação experimental e numérica. Escola de Engenharia da Universidade do Minho, Guimarães, Portugal, 2003. 
ENGENHARIA CONCRETA. Fibra para concreto: Principais tipos de fibras e utilização, 2016. Disponível em: $<$ https://engenhariaconcreta.com/fibra-para-concretoprincipais-tipos-de-fibras-e-utilizacao/>. Acesso em 07/08/2019.

KANG, S. T.; LEE B.Y.; KIM J.K.; KIM, Y.Y. The effect of fibre distribution characteristics on the flexural strength of steel fibre reinforced ultra high strength concrete: Construction and Building Materials, 2011.

Lee, Y.; Kang, S.; Kim, J. Pullout behavior of inclined steel fiber in an ultra-high strength cementitious matrix. Construction and Building Materials, 2010.

LIMA, R.P. Utilização de fibras metálicas com funções estruturais em estruturas de betão. Faculdade de engenharia da Universidade do porto, Portugal, 2012.

MORAES, N. Comportamento à Punção de Lajes Lisas em Concreto Reforçado com Fibras de Aço sob Carregamento Simétrico. 343 p. Universidade de Brasília, Brasília, 2013.

MINELLI, Fausto.; CONFORTI, A.; CUENCA, E.; PLIZZARI, Giovanni. Are steel fibres able to mitigate or eliminate size effect in shear?. s.I: Materials and Structures, v, 47, p. 459-473, 2014.

POH, J.; TAN, K.H.; PETERSON, G.L.; WEN, D. Structural testing of steel fibre reinforced concrete (SFRC) tunnel lining segments in Singapore. Land Transport Authority, Hampshire Road, ITA 2008.

PUJADAS, P.; BLANCO, A.; CAVALARO, S.; AGUADO, A. Plastic fibres as the only reinforcement for flat suspended slabs: Experimental investigation and numerical simulation, Construction and Building Materials, Vol. 57, 2014.

PUJADAS, P.; BLANCO, A.; FUENTE, A.; AgUADO, A. Cracking behavior of FRC slabs with traditional reinforcement. Materials and Structures, v.45, 2012.

RILEM TC 162 - TDF. Test and design methods for steel fibre reinforced concrete: Bending test, Final Recommendation. Materials and Structures, v. 35, p. 579-582, 2002.

TIBERTI, Giuseppe.; MINELLI, Fausto.; PLIZZARI, Giovanni. Reinforcement optimization of fiber reinforced concrete linings for conventional tunnels. Itália: Composites: Part B, v. 58, p. 199-207, 2014. 